\title{
Negative SPR Signals During Low Molecular Weight Analyte Recognition
}

H. Bonnet, L. Coche-Guérente, E. Defrancq, N. Spinelli, A. Van der Heyden, J. Dejeu*

Univ. Grenoble Alpes, CNRS, DCM UMR-5250, F-38000 Grenoble, France

Corresponding author : Jérôme Dejeu, + 336873978 84, jerome.dejeu@ univ-grenoble-alpes.fr

SPR response monitored during the aptasensor build-up 2

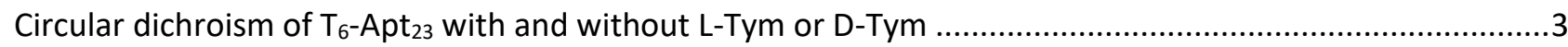

QCM-D responses monitored during the aptasensor build-up ...........................................................

Sensorgram recorded during the $\mathrm{D}-\mathrm{Tym}$ injection on $\mathrm{T}_{6}-\mathrm{Apt}_{23}$ monolayer.............................................

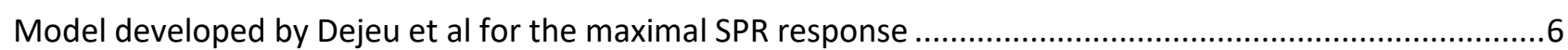




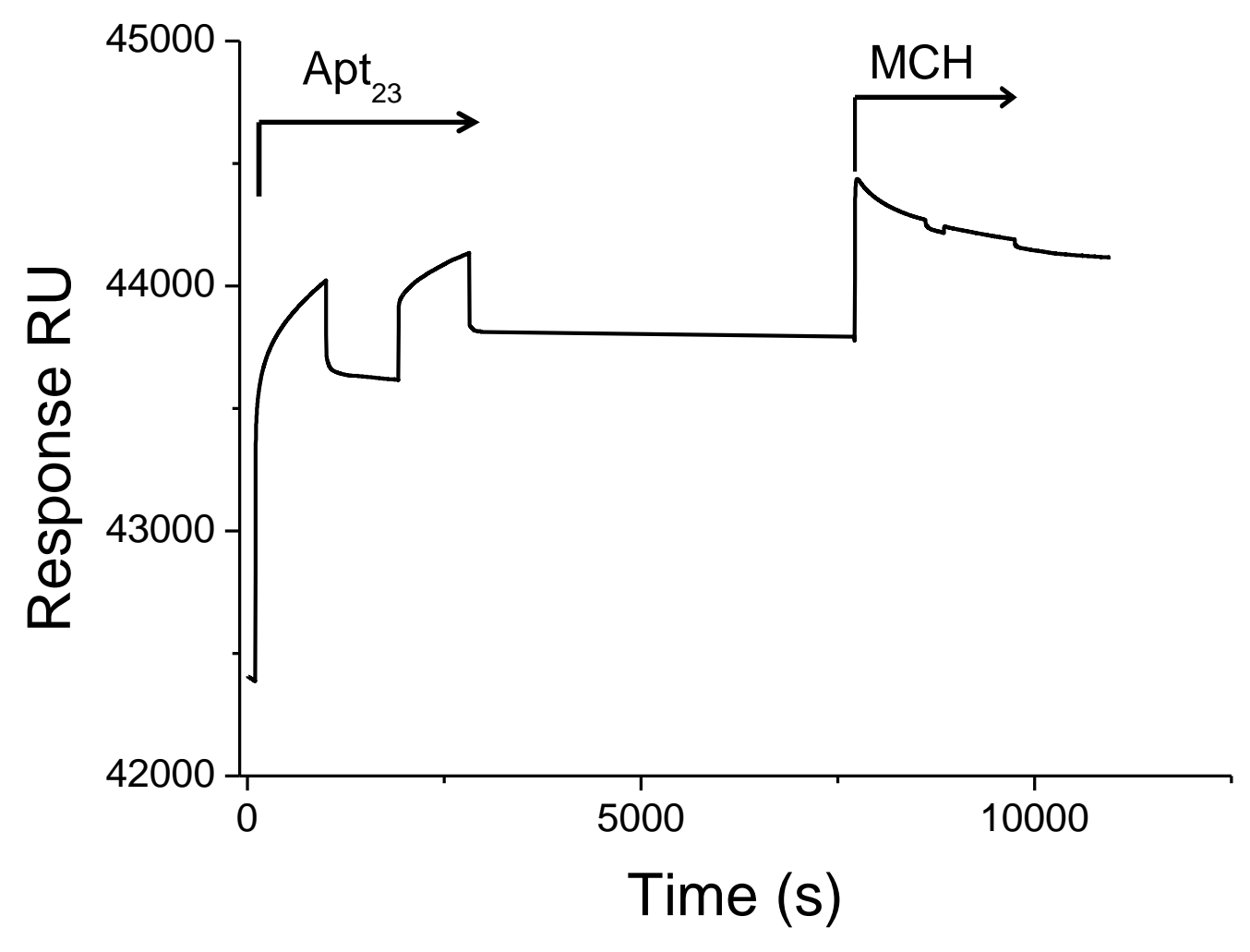

Figure SI-1: Sensorgram recorded during the build-up of the aptasensor on the gold surface. Thiolated 23-mer aptamer $(1 \mu \mathrm{M})$ was injected followed by $\mathrm{MCH}(1 \mathrm{mM})$ injection. $\mathrm{T}=25^{\circ} \mathrm{C}$. Flow rate: $2 \mu \mathrm{L} / \mathrm{min}$ 
Circular dichroism of $\mathrm{T}_{6}-\mathrm{Apt}_{23}$ with and without L-Tym or D-Tym

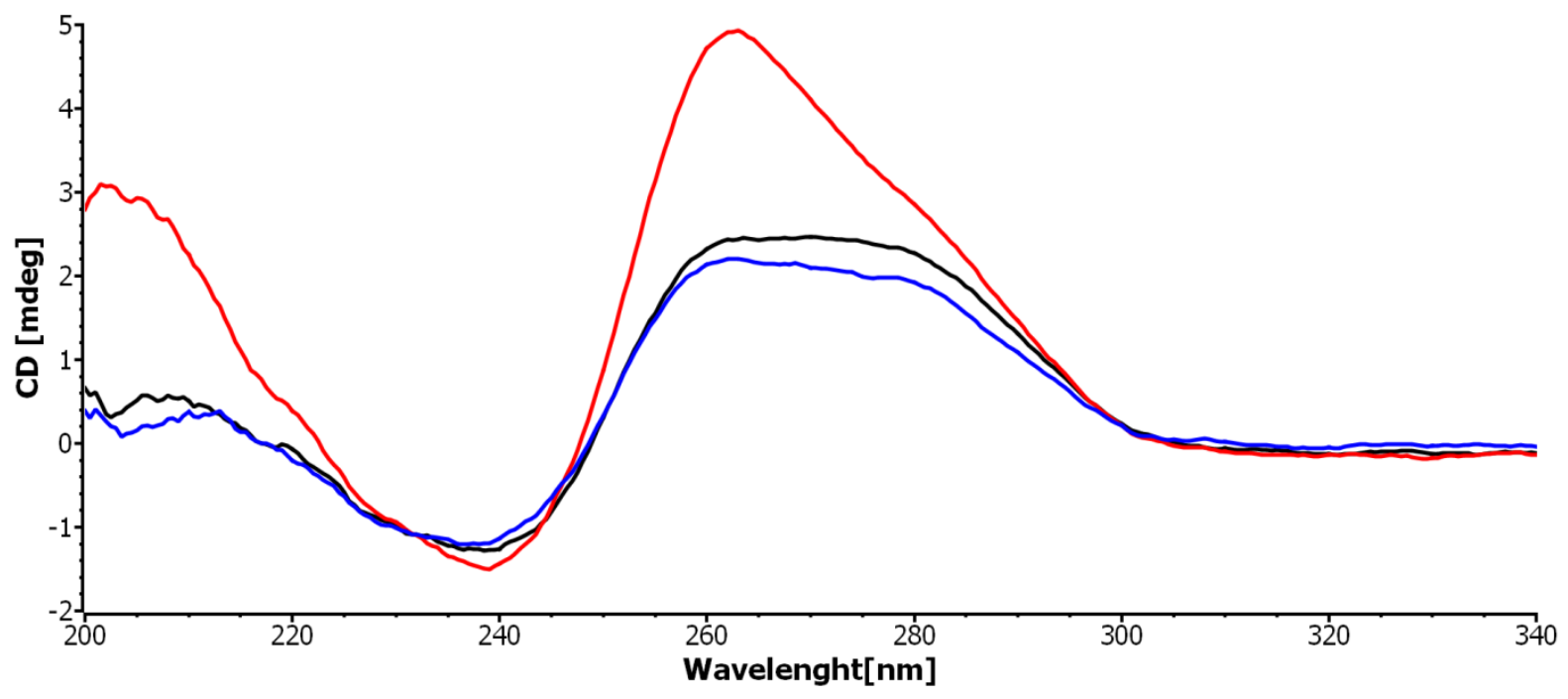

Figure SI-2: CD spectra of $\mathrm{T}_{6}-\mathrm{Apt}_{23}$ with and without L-Tym or D-Tym $(15 \mu \mathrm{M}$ in $5 \mathrm{mM}$ Tris, 10 $\mathrm{mM} \mathrm{MgCl}_{2}, 50 \mathrm{mM} \mathrm{NaCl}$ ). (black line) T6-Apt23; (red line) $\mathrm{T}_{6}-\mathrm{Apt}_{23}+\mathrm{L}-\mathrm{Tym}$ (leq), (blue line) $\mathrm{T}_{6-}$ $\mathrm{Apt}_{23}+\mathrm{D}$-Tym (1eq) 


\section{QCM-D responses monitored during the aptasensor build-up}

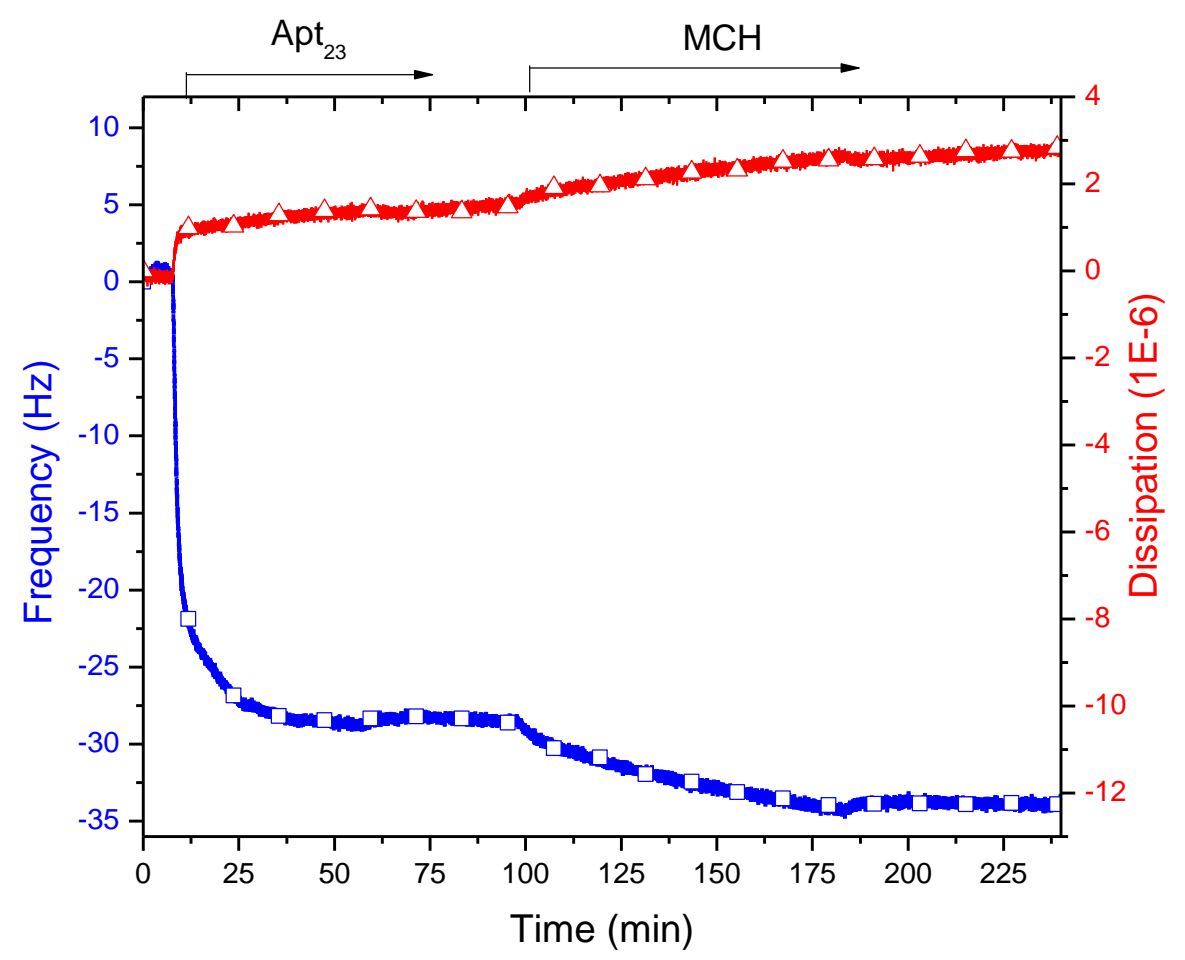

Figure SI-3: QCM-D profile characterizing the build-up of an aptasensor on the quartz gold sensor. Thiol aptamer $(1 \mu \mathrm{M})$ was injected during fixed duration followed by $\mathrm{MCH}(1 \mathrm{mM})$. The frequency shift and the dissipation shift are represented for overtones: $i=7$ ( $\square$ blue line for frequency and $\Delta$ red line for dissipation). Start and duration of aptamer and $\mathrm{MCH}$ injections are indicated by arrows on top of the profile. Before sample injection and after saturated binding, the surfaces were exposed to pure running buffer. Flow rate: $50 \mu \mathrm{L} / \mathrm{mL}$. 
Sensorgram recorded during the D-Tym injection on $\mathrm{T}_{6}-\mathrm{Apt}_{23}$ monolayer

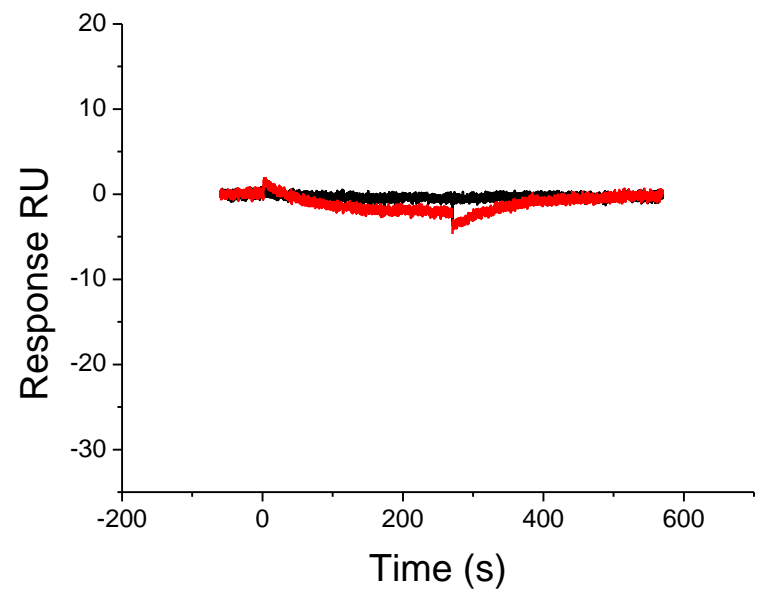

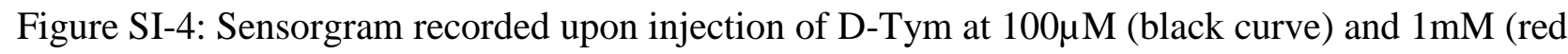
curve) on the active flowcell after double subtraction procedure. 


\section{Model developed by Dejeu et al for the maximal SPR response}

See reference 4 below for further details.

\section{- Refractive Index Increment correction:}

Classically, the molar refractive index increment of the complex analyte/ligand can be defined from the RII of both the analyte and the ligand ones by equation (SI-1):

$$
\left(\frac{d n}{d c}\right)_{L A}=\left(\frac{d n}{d c}\right)_{L}+V \cdot\left(\frac{d n}{d c}\right)_{A}
$$

The L-Tym/aptamer interaction having a 1:1 stoichiometry, ${ }^{3} \mathrm{~V}=1$ in our study

Considering a deviation factor for the RII of the analyte/ligand complex, eq (SI-1) is not valid anymore and a correction term has to be included. As a consequence, the RII of the L-Tym/aptamer complex is expressed as a function of the RII of the aptamer by introducing a correction factor, $\mathrm{x}$ :

$$
\left(\frac{d n}{d c}\right)_{\text {correction }}=x\left(\frac{d n}{d c}\right)_{L}
$$

where $(\mathrm{dn} / \mathrm{dc})_{\text {correction }}$ is the correction of the L-Tym/aptamer complex RII defined as a function of the RII of the aptamer. By adding this correction factor to the sum of the RII of the two entities separately considered eq ((SI-1)), we obtain eq (SI-3):

$$
\left(\frac{d n}{d c}\right)_{L A}=\left(\frac{d n}{d c}\right)_{L}+V \cdot\left(\frac{d n}{d c}\right)_{A}+\left(\frac{d n}{d c}\right)_{\text {correction }}=(1+x)\left(\frac{d n}{d c}\right)_{L}+V \cdot\left(\frac{d n}{d c}\right)_{A}
$$


- Dejeu's Formula ${ }^{4-5}$

$R U_{A_{\max }}=R U_{L}\left(\frac{1}{\rho} \frac{1-e^{-\frac{\rho d_{L}}{d_{p}}}}{1-e^{-\frac{d_{L}}{d_{p}}}}(\beta \times V+1+x)-1\right)$

Where $\mathrm{RU}_{\mathrm{Amax}}$ is the expected maximum response at a single site, $\mathrm{RU}_{\mathrm{L}}$ is the aptamer immobilization level, $d p$ is the effective penetration depth of the SPR wave $(175 \mathrm{~nm}), d_{L}$ and $d_{L A}$ are the thicknesses before and during the interaction respectively, $\rho$ the folding ratio of the aptamer $\left(\rho=\mathrm{d}_{\mathrm{LA}} / \mathrm{d}_{\mathrm{L}}\right), \quad \beta=M W_{A} \cdot(d n / d c)_{A}^{\prime} / M W_{L} \cdot(d n / d c)_{L}^{\prime}$ is the ratio of the mass-weighted RII of the analyte versus the ligand and the $\mathrm{V}$, the valency i.e. the ratio number of analytes per number of ligands involved in the recognition ( $\mathrm{V}=1$ in the present system). $\mathrm{MW}_{\mathrm{A}}$ and $\mathrm{MW}_{\mathrm{L}}$ are the molecular weights of the injected analyte (L-Tym, $180.2 \mathrm{~g} \cdot \mathrm{mol}^{-1}$ ) and of the immobilized ligand (aptamer, 9182 g. $\left.\mathrm{mol}^{-1}\right)$, respectively. $(d n / d c)_{A}^{\prime}$ and $(d n / d c)_{L}^{\prime}$ are the refractive index increments (RII) for the analyte (L-Tym, $\left.0.215 \mathrm{~cm}^{3} \cdot \mathrm{g}^{-1}\right)^{1}$ and for the ligand (aptamer, $0.238 \mathrm{~cm}^{3} \cdot \mathrm{g}^{-1}$ ) respectively. 


\section{Reference}

1. Vianini, E.; Palumbo, M.; Gatto, B. Bioorg. Med. Chem. 2001, 9, 2543-2548.

2. Rich, R. L.; Myszka, D. G. Advances in Surface Plasmon Resonance Biosensor Analysis. Curr. Opin. Biotechnol. 2000, 11, 54-61.

3. L. Challier, R. Miranda-Castro, B. Barbe, C. Fave, B. Limoges, E. Peyrin, C. Ravelet, E. Fiore, P. Labbé, L. Coche-Guérente, E. Ennifar, G. Bec, P. Dumas, F. Mavré, V. Noël. Multianalytical Study of the Binding between a Small Chiral Molecule and a DNA Aptamer: Evidence for Asymmetric Steric Effect upon 3'- versus 5'-End Sequence Modification, Anal. Chem., 2016, 88, 11963-11971.

4. J. Dejeu, H. Bonnet, N. Spinelli, E. Defrancq, L. Coche-Guerente, A. Van der Heyden, P. Labbé, Impact of conformational transitions on SPR signals-Theoretical treatments and application in small analytes/aptamer recognition, J. Phys. Chem. C., 2018, 122, 21521 21530.

5. Osypova, A.; Thakar, D.; Dejeu, J.; Bonnet, H.; Van der Heyden, A.; Dubacheva, G. V.; Richter, R. P.; Defrancq, E.; Spinelli, N.; Coche-Guérente, L. et al. Sensor Based on Aptamer Folding to Detect Low-Molecular Weight Analytes. Anal. Chem. 2015, 87, 75667574. 